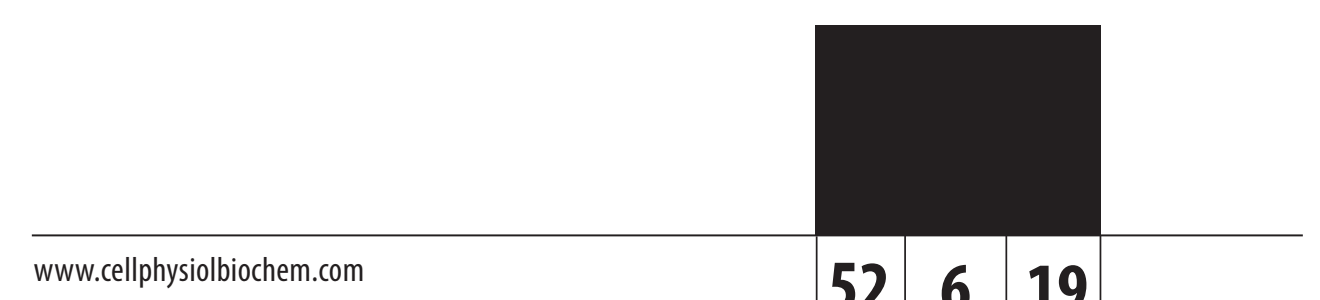

\title{
Cellular Physiology
}

\section{Original and Biochemistry}

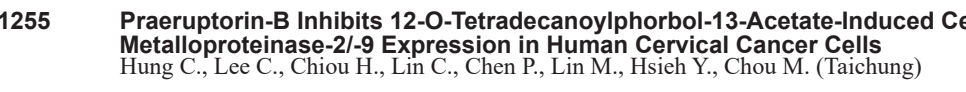

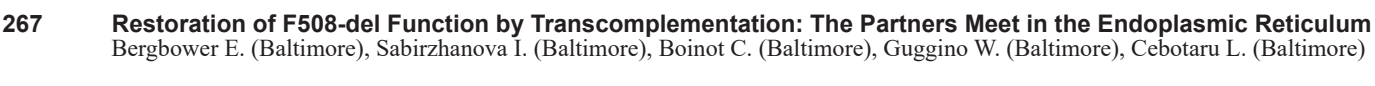

1280 Exosomes Treatment Mitigates I Ischemic Brain Damage but Does Not Improve Poststroke Neurological Outcome

(P). Peoria). Veeravalli K. (Pooria

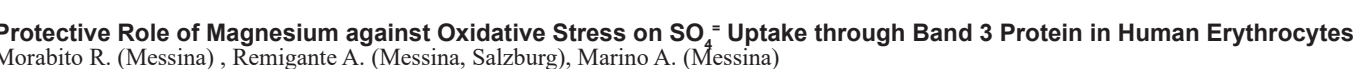

1309 In Vitro Grown Micro-Tissues for Cardiac Cell Replacement Therapy in Vivo

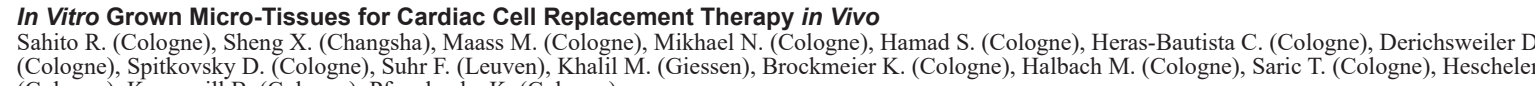

ROS Generated by Upconversion Nanoparticle-Mediated Photodynamic Therapy Induces Autophagy via PI3K/AKT/ mTOR Signaling

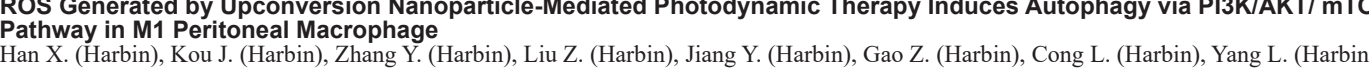

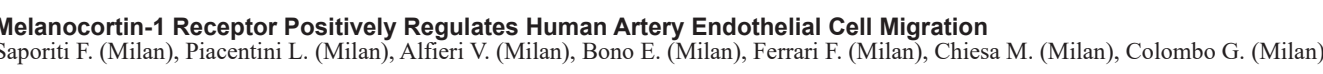

BDNF and NT3 Reprogram Human Ectomesenchymal Dental Pulp Stem Cells to Neurogenic and Gliogenic Neural Crest Progenitors

Ouabain Modulates the Adherens Junction in Renal Epithelial Cells

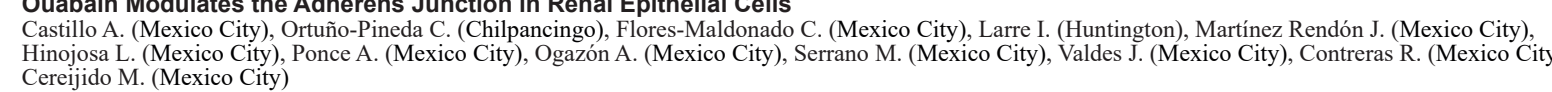

Vistatin Promotes Monocye Adhesion by Upregulating ICAM-1 and VCAM-1 Expression in Endothella Cells via Activation of

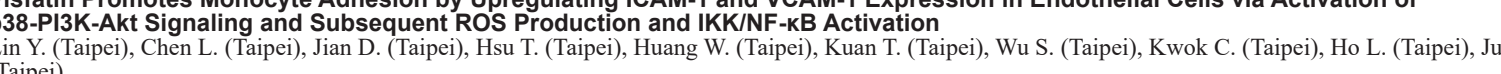

The microRNA miR-3755-3p and the Tumor Suppressor NDRG2 are Involved in Sporadic Amyotrophic Lateral Sclerosis
Rohm M. (Bochum), May C. Bochum), Marcus K. (Bochum), Steinbach S. (Bochum), Theis V. (Bochum), Theiss C. (Bochum), Matschke V. (Bochum

Regulation of Plasma Membrane Localization of the Nat-Taurocholate Co-Transporting Polypeptide by Glycochenodeoxycholate and Tauroursodeoxycholate
Mayer P. (Diisseldorf), Qvartshava N. (Diisseldorf), Sommerfeld A. (Disseldorf), Görg B. (Disseldorf), Häussinger D. (Diisseldorf)

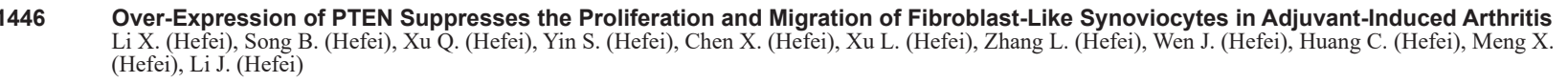

Adipose-Derived Mesenchymal Stromal Cells Under $H$ Hod

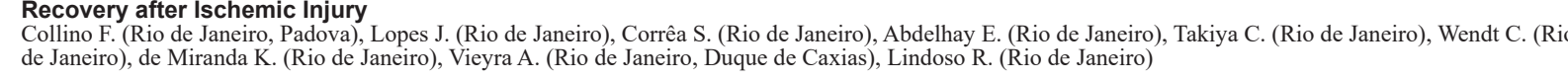

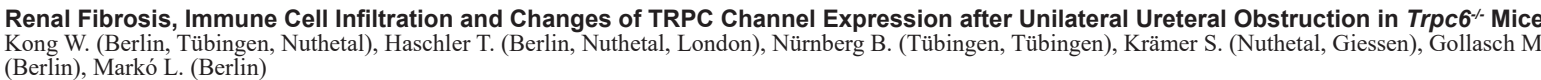

ZNF281 Regulates Cell Proliferation, Migration and Invasion in Colorectal Cancer through Wnt/B-Catenin Signaling
Qin C. (Kaifeng), Bu P. (Kaifeng), Zhang Q. (Kaifeng), Chen J. (Kaifeng), Li Q. (Kaifeng), Liu J. (Kaifeng), Dong H. (Kaifeng), Ren X. (Kaifeng)

Cadmium Induces Migration of Colon Cancer Cells: Roles of Reactive Oxygen Species, P38 and Cyclooxygenase-2
Naji S. (Beirut), Isa K. (Berint), Eid A. (Beirut), Irathi R. (Al-Ain), Eid A. (Beirut, Doha)

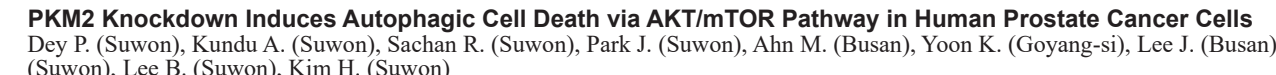

ADAMTS1 Promotes Adhesion to Extracellular Matrix Proteins and Predicts P
Tan I. (Adelaide), Frewin K. (Adelaide), Ricciardelli . (Adelaide), Russell D. (Addelaide)

Kim N. (Busan), Kim I.

Developmental Retinal Angiogenesis by Vessel Regressio

Erratum

Erratum

Erratum

Erratum

Erratum

Erratum

Cover illustration

In Vitro Grown Micro-Tissues for Cardiac Cell Replacement Therapy in Vivo. See Original by Sahito, et al.
in Cell Physiol Biochem 2019;52:1309-1324.

\section{Cellular Physiology and Biochemistry}

International Journal of

Experimental Cellular Physiology, Biochemistry and Pharmacology
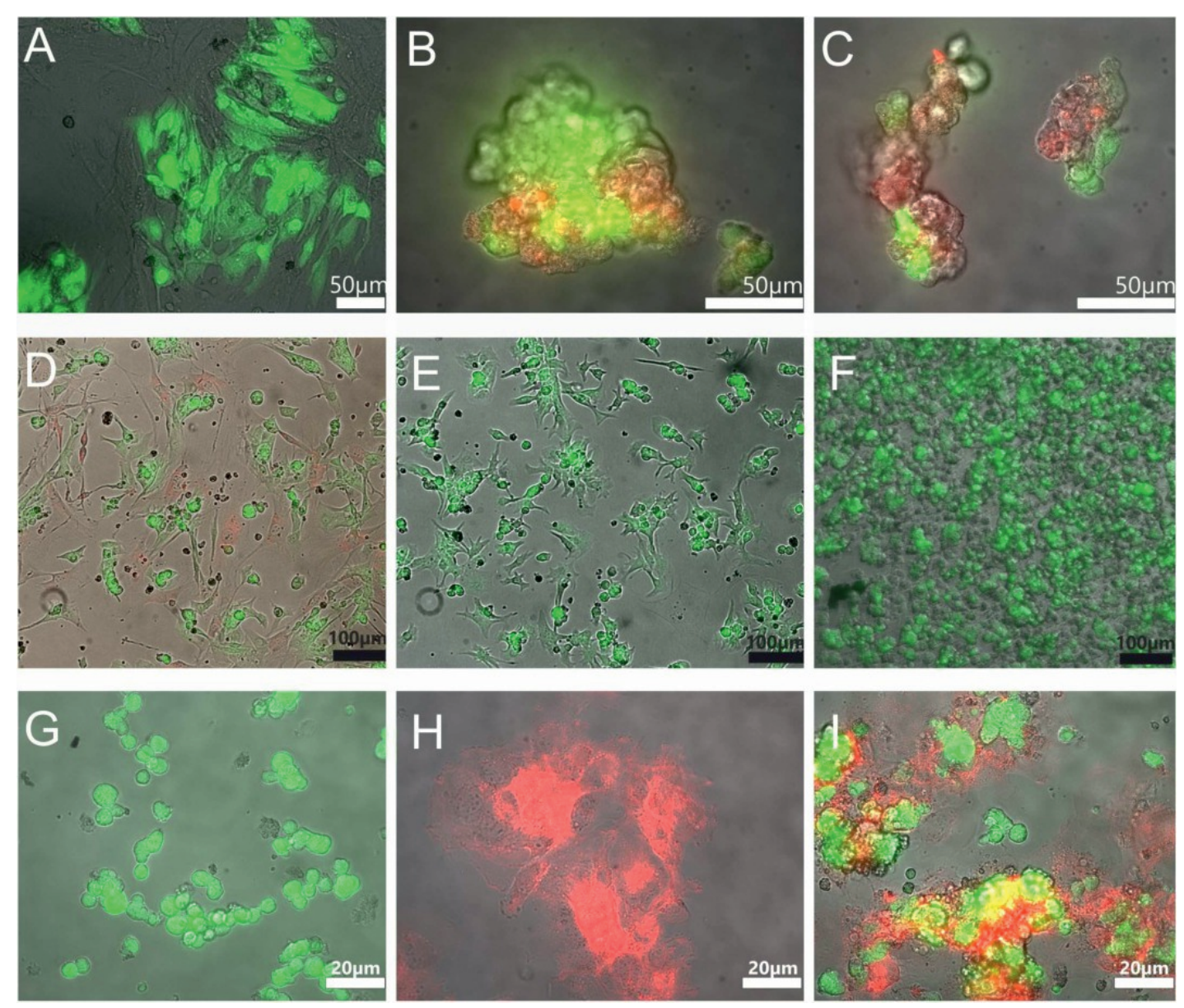

Open access 